\title{
Influence of surface treatment on the magnetic properties of $\mathrm{Ga}_{x} \mathbf{M n}_{1-x} \mathrm{As}$ thin films
}

\author{
F. Maccherozzi, ${ }^{1}$ G. Panaccione, ${ }^{1}$ G. Rossi, ${ }^{1,2}$ M. Hochstrasser, ${ }^{3}$ M. Sperl, ${ }^{4}$ M. Reinwald, ${ }^{4}$ G. Woltersdorf, ${ }^{4}$ W. Wegscheider, ${ }^{4}$ \\ and C. H. Back ${ }^{4}$ \\ ${ }^{1}$ Laboratorio Nazionale TASC, INFM-CNR, in Area Science Park, S.S.14, Km 163.5, I-34012, Trieste, Italy \\ ${ }^{2}$ Dipartimento di Fisica, Univ. di Modena e Reggio Emilia, Via A. Campi 213/A, I-41100, Modena, Italy \\ ${ }^{3}$ Laboratorium für Festkörperphysik, Wolfgang-Pauli-Strasse 16, ETH Hönggerberg, CH-8093 Zürich, Switzerland \\ ${ }^{4}$ Institut für Experimentelle Physik, Univ. Regensburg, D-93040 Regensburg, Germany \\ (Received 11 April 2006; revised manuscript received 7 June 2006; published 28 September 2006)
}

\begin{abstract}
We present a surface science study on carefully characterized $\mathrm{Ga}_{x} \mathrm{Mn}_{1-x}$ As films grown on $\mathrm{GaAs}(100)$, where we investigate the influence on magnetic properties of different surface cleaning procedures. X-ray magnetic circular dichroism reveals a net remnant magnetization along the (100) axis, after chemical etching is performed. The analysis of Mn $L_{2,3}$ XAS line shapes in different environment indicates that $\mathrm{Mn}$ in $\mathrm{Ga}_{x} \mathrm{Mn}_{1-x} \mathrm{As}$ behaves as a bulk impurity. By exploiting the different depth sensitivity between x-ray photoemission and x-ray absorption we observe the presence of a Mn depleted layer at the surface after etching.
\end{abstract}

DOI: 10.1103/PhysRevB.74.104421

PACS number(s): 75.50.Pp, 78.70.Dm, 81.40.Rs, 75.70.-i

\section{INTRODUCTION}

$\mathrm{Ga}_{x} \mathrm{Mn}_{1-x} \mathrm{As}$ is often seen as the most promising diluted ferromagnetic semiconductor (DMS) for future spintronics devices. ${ }^{1,2}$ The large research effort on $\mathrm{Ga}_{x} \mathrm{Mn}_{1-x}$ As makes it a prototypical system of a DMS. The incorporation of Mn in GaAs above the solubility limit, but avoiding undesirable phase separation, produces a system that contains local magnetic moments on the Mn sites and the required concentration of holes that enables the interaction between Mn spins. ${ }^{3}$ Driven by the theoretical prediction of stable ferromagnetic (FM) properties even above room temperature, ${ }^{1}$ the synthesis of $\mathrm{Ga}_{x} \mathrm{Mn}_{1-x}$ As has been optimized by low temperature molecular beam epitaxy (MBE) growth followed by postgrowth annealing. The Curie temperature $\left(T_{c}\right)$ value, since the discovery of FM in the early preparations of $\mathrm{Ga}_{x} \mathrm{Mn}_{1-x} \mathrm{As}$, made in 1996 by Ohno et al., ${ }^{4}$ has increased, but the newer materials are rich of defects. Interstitial Mn and $\mathrm{As}_{\mathrm{Ga}}$ antisites, both acting as a double donor, hinder the full contribution to FM of the free holes provided by substitutional $\mathrm{Mn}^{5,6}$ The Mn defects are now understood as the main responsible of the reduction of $T_{c}$ observed when increasing the film thickness as well as when capping with GaAs. ${ }^{7-9}$ Moreover, the importance of diffusion of the highly mobile interstitial Mn to the surface, and the passivation of Mn due to oxidation have been recently proposed. ${ }^{6,10,11}$

Concerning the spectroscopic investigation of $\mathrm{Ga}_{x} \mathrm{Mn}_{1-x} \mathrm{As}$ magnetic properties, the presence of two inequivalent $\mathrm{Mn}$ sites and the difficult estimate, due to different sample preparation, of the undesired interstitial Mn content has often led to contradictory results, suggesting in some cases a marked reduction of the magnetic moments.,12-14 Recent $\mathrm{X}$-ray absorption (XAS) and x-ray magnetic dichroism (XMCD) results by Edmonds et al. show (i) large $\mathrm{Mn}$ moments when forcing spins along the hard magnetization axis in a magnetic field of $2 \mathrm{~T},{ }^{11-15}$ and (ii) the importance of anisotropy and hybridization with the host valence bands. ${ }^{16}$ Furthermore, by the analysis of the XAS line shapes the authors ascribe the previously reported "magnetization deficit" to the measure of the surface Mn oxides instead of the truly FM substitutional Mn. ${ }^{7,12}$ The relationship between sample preparation conditions and disorder/diffusion effects has considerable implications on the understanding of the magnetic properties of $\mathrm{Ga}_{x} \mathrm{Mn}_{1-x}$ As. This detailed understanding is of fundamental importance for establishing realistic conditions for the realization of future devices and for the control of their magnetic functionalities. In order to obtain reliable data it is then mandatory (i) to carefully characterize the samples and (ii) to adopt chemical sensitive experimental techniques capable to probe the local magnetic moments and to distinguish surface from bulk electronic properties.

In this paper we report XMCD, XAS, and X-ray photoemission spectroscopy (XPS) results of carefully characterized $\mathrm{Ga}_{x} \mathrm{Mn}_{1-x}$ As films grown on $\operatorname{GaAs}(100)$. XMCD experiments reveal a net magnetization along the (100) axis only after removal of the native oxides by means of chemical etching. An intense XMCD signal is measured in remnant field, in good qualitative agreement with the data measured by Edmonds et al. in a large magnetic field applied along the hard magnetization axis. ${ }^{15}$ The XPS analysis reveals a Mn depleted layer at the surface after etching. The Mn $L_{2,3}$ XAS line shapes indicate that $\mathrm{Mn}$ in $\mathrm{Ga}_{x} \mathrm{Mn}_{1-x} \mathrm{As}$ behaves as an impurity, with a sizeable degree of correlation and localization. The sputtering and annealing procedures also influence the chemical composition at the surface and in the bulk, as well as the magnetic properties of Mn.

\section{EXPERIMENTAL: SAMPLE PREPARATION AND CHARACTERIZATION}

The $\mathrm{Ga}_{x} \mathrm{Mn}_{1-x}$ As diluted samples were grown on (100) oriented GaAs substrates. The alloy parameter $x$ ranges between 0.021 and 0.037 . One representative sample with $x$ $=0.024$ was fractured in three pieces $(S 1, S 2, S 3)$ that were treated differently: $S 1$ was left "as received," i.e., without post-growth treatments, but aged in atmospheric pressure, $S 2$ was etched, $S 3$ was etched, and then $\mathrm{Ar}^{+}$ion sputtered and annealed in situ, in ultrahigh vacuum (UHV). 


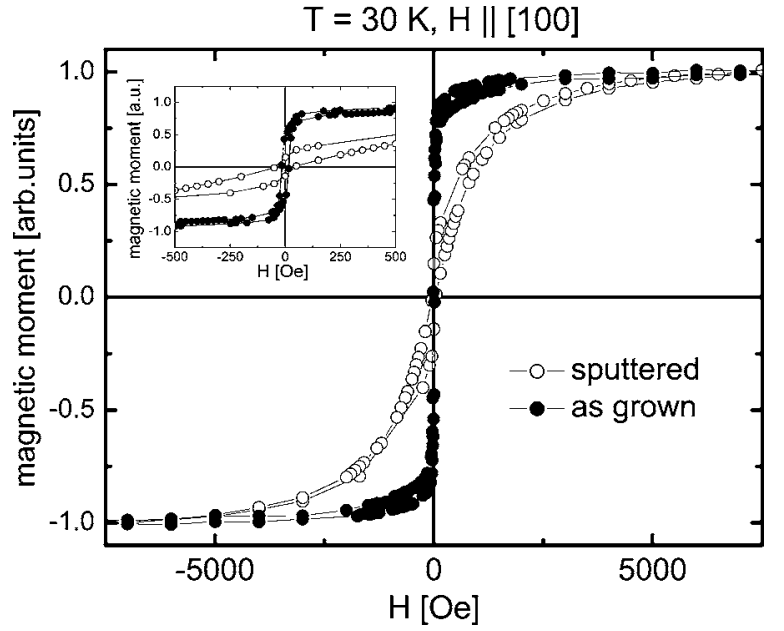

FIG. 1. SQUID magnetization loops with the magnetic field applied along the (100) easy magnetization axis measured at $30 \mathrm{~K}$. Full circles show the loop of the as grown sample. Open circles show the loop after surface preparation. The inset shows a zoom into the low field region.

Ferromagnetic samples have been produced at lowtemperature growth conditions as described below: prior to $(\mathrm{Ga}, \mathrm{Mn})$ As growth, a $300 \mathrm{~nm}$ GaAs buffer was grown at standard conditions [substrate temperature $T_{s}=600^{\circ} \mathrm{C}$; arsenic flux $\approx 1 \times 10^{-5}$ Torr beam equivalent pressure (BEP), III-V ratio $\approx 20$ (BEP), GaAs growth rate $\approx 2.8 \AA / \mathrm{s}]$. Subsequently, the sample temperature was reduced to $260{ }^{\circ} \mathrm{C}$. Meanwhile the temperature of the gallium effusion cell was reduced, resulting in a GaAs growth rate of $\approx 0.7 \AA / \mathrm{s}$. The arsenic flux was maintained constant. After the substrate temperature had stabilized, $20-50 \mathrm{~nm} \mathrm{Ga}_{1-x} \mathrm{Mn}_{x} \mathrm{As}$ was grown with a manganese content $x \approx 2-4 \%$. The crystal quality of the epilayer was monitored in situ via reflection high-energy electron diffraction (RHEED). The manganese concentration was measured ex situ by XPS and magnetic properties were measured by a superconducting quantum interference device (SQUID) (see Fig. 1). Some of the (Ga, Mn)As samples have been annealed ex situ at $180{ }^{\circ} \mathrm{C}$ in air for about $60 \mathrm{~h}$.

Chemical etching is a very efficient way to remove the surface Mn oxide layer, as reported in Ref. 10. Samples $S 2$ and $S 3$ have been treated by etching the "as received" epitaxial films in pure $\mathrm{HCl}$ for a few seconds, then rinsed in deionized water and inserted in UHV (base pressure 4 $\times 10^{-10}$ mbar). The XPS and Auger spectra of etched samples reveal a surface rich in carbon and oxygen, while the $\mathrm{Mn}$ is reduced to trace level. The characterized samples were transferred in UHV to the main chamber, where the sample holder is fitted on a liquid helium flow cryostat, capable of refrigerating the sample to $17 \mathrm{~K}$. In the case of sample $S 3$ the etching was followed in situ by $\mathrm{Ar}^{+}(750 \mathrm{eV}$, 5 mins, sample current $1 \mu \mathrm{A}$ ) sputtering, at an estimated sputtering rate of $1 \mathrm{ML} / \mathrm{min}$, assuming unity sputtering yield. After sputtering, no trace of oxygen was detected in Auger spectra, whereas residual carbon and an intense Mn peak were measured. The sample was then annealed at $200{ }^{\circ} \mathrm{C}$ in an oxygen pressure of $7 \times 10^{-7} \mathrm{mbar}$ for $3 \mathrm{~h}$.
The experiments were performed at the Advanced Photoemission Experiment (APE) INFM undulator beamline at the Elettra Synchrotron radiation facility in Trieste, delivering both linearly and circularly polarized light. ${ }^{17} \mathrm{Mn} L_{2,3}$ XAS and XMCD measurements were obtained by measuring the sample drain current with an energy resolution of $150 \mathrm{meV}$ and a circular polarization rate of $70 \%$. The photon incidence angle was kept fixed at $45^{\circ}$ from the sample surface normal, as indicated in Fig. 2. Both XPS and XAS/XMCD measurements were possible at identical sample positions (see the inset of Fig. 2). XPS data have been acquired by a sevenchannel Omicron EA-125 analyzer at normal emission using linearly polarized $\mathrm{x}$ rays with photon energies of 220 and $880 \mathrm{eV}$. The overall energy resolution (photons + analyzer) was about $200 \mathrm{meV}$. In both photoemission and photoabsorption measurements the $\mathrm{x}$-ray beam size on the sample surface was of the order of $100 \times 200 \mu \mathrm{m}^{2}$. The samples were magnetized by applying a magnetic field using a horseshoe magnet able to deliver up to 2000 Oe in pulsed mode along the sample surface plane, i.e., the magnetic field is applied along the (100) easy magnetization axis. All XMCD measurements were performed in remnant magnetization, by reversing the sign of the field pulse before each scan, and keeping fixed the photon helicity. Fully consistent results were obtained with opposite photon helicity. We did not observe any dependence of the XAS spectral shape with respect to temperature, Mn concentration, and substrate orientation.

\section{RESULTS}

The Mn $L_{2,3}$ XAS spectra are presented as normalized to the incoming flux $\left(I_{0}\right)$, in Fig. 2 . The proper normalization was obtained by measuring in identical conditions a clean $\operatorname{GaAs}(100)$ surface and using this spectrum to filter all substrate contributions from the Mn spectra. The Mn $L_{2,3}$ transition to the continuum were simulated by a standard step function.

The data from the $S 1$ (as-grown) and $S 2$ (etched) samples, as measured at room temperature, are compared to each other in Fig. 2(a). $S 1$ displays the characteristic structures of the atomic $\mathrm{Mn}^{2+}$ state, typical of the Mn oxides, with well defined structures at 638 (L3 peak), 639.3, and $641.5 \mathrm{eV}$. The $S 2$ spectrum is nearly free of structures in between the edges, and displays two broad shoulders around 639.1 and $641.2 \mathrm{eV}$. The $L_{3}$ peak is centered at $637.6 \mathrm{eV}$, i.e., with an energy shift of $0.4 \mathrm{eV}$ with respect to the spectrum of $S 1$. Panel (b) of Fig. 2 presents the Mn $L_{2,3}$ XAS spectra of $S 2$ and of other metallic Mn alloys, namely, a thin (8 ML) epitaxial MnPt film grown on $\mathrm{Fe}(100)$, and a thick MnAs single crystal along with the spectrum of pure Mn (thick film). All XAS spectra have been obtained in identical experimental conditions, with the sample maintained at room temperature. We observe that the line shape of the Mn XAS in $\mathrm{Ga}_{x} \mathrm{Mn}_{1-x} \mathrm{As}$ and PtMn present strong similarities, with the characteristic double peak at the $L_{2}$ edge, and a weak shoulder at $641.2 \mathrm{eV}$. The branching ratio $B$ defined as $B$ $=\left(A_{2 p 3 / 2}\right) /\left(A_{2 p 3 / 2}+A_{2 p 1 / 2}\right)$, where $A$ is the integrated area under the peaks, after normalization and background subtrac- 

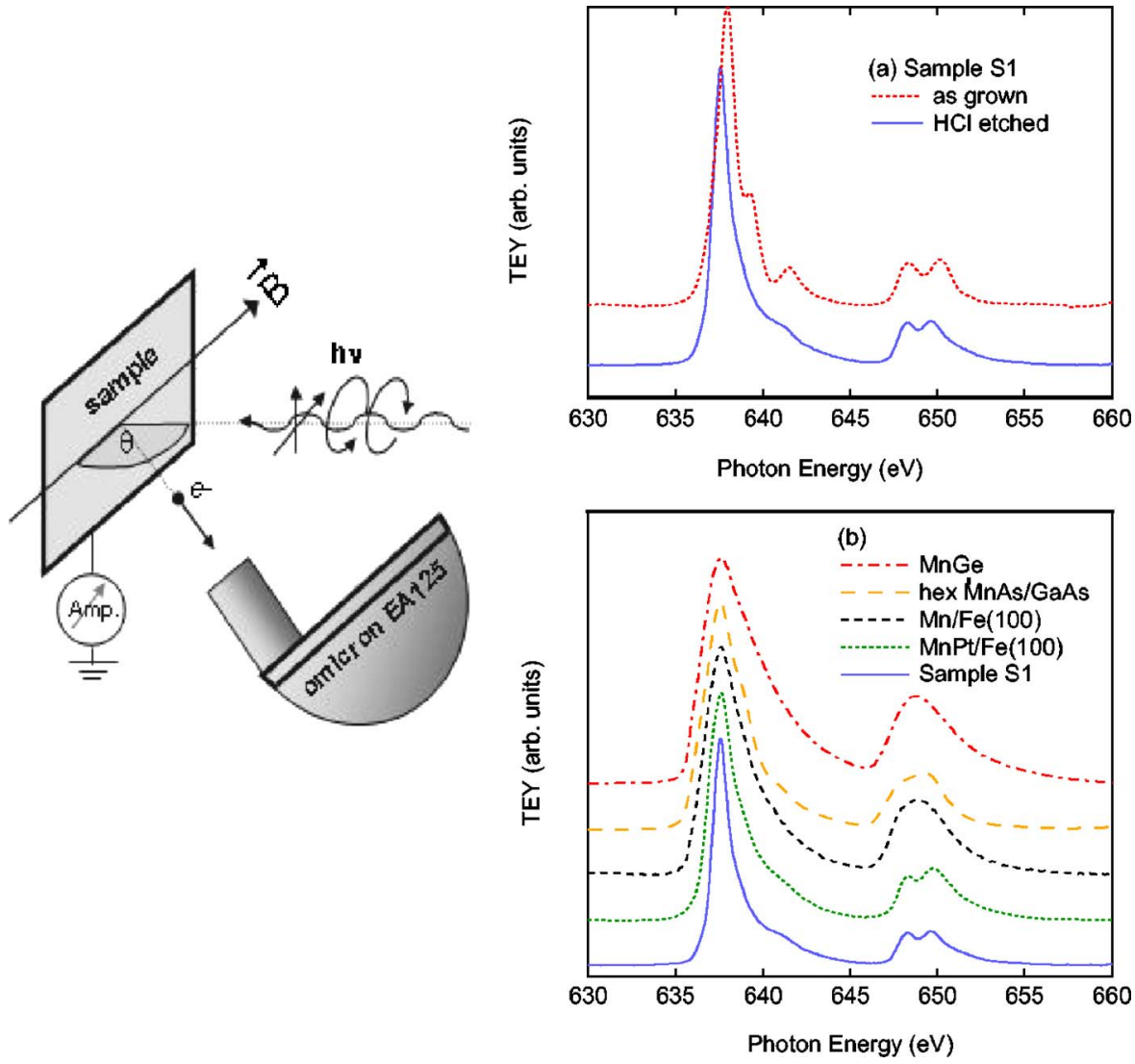

FIG. 2. (Color online) Left: Sketch of the experimental geometry for XPS/XAS/XMCD measurements. Right, Panel (a): Mn $L_{2,3}$ XAS spectra at room temperature of samples $S 1$ as-grown (dashed line) and sample $S 2$ after etching (continuous line). Panel (b): sample $S 2$ etched (continuous line) compared with bcc $\operatorname{MnPt}(10 \AA) / \mathrm{Fe}(100) \quad$ (green dashed line), metallic $\mathrm{Mn} / \mathrm{Fe}(100)$ (black dotted line), hexagonal MnAs/GaAs(100) (yellow dashed-dotted line), and $\mathrm{Mn} / \mathrm{Ge}$ alloy (red dot dashed lined). All spectra have been normalized to unity for comparison. tion, is 0.76 for PtMn, 0.79 for $\mathrm{Ga}_{x} \mathrm{Mn}_{1-x} \mathrm{As}$, and 0.66, i.e., the statistical value, for metallic $\mathrm{Mn}$.

Figure 3 presents the magnetization dependent XAS spectra of sample $S 2$ measured in remnant magnetization at $17 \mathrm{~K}$, together with the difference spectrum (XMCD). The sample was magnetized by external field pulses of \pm 1 KOe along the (100) easy magnetization axis. The line shape of the XMCD is quite similar to those previously reported in Refs. 10-15, with small differences in the preedge region. The XMCD asymmetry, defined as $\left(I_{M_{+}}-I_{M_{-}}\right) /\left(I_{M_{+}}+I_{M_{-}}\right)$at the $L_{3}$ peak

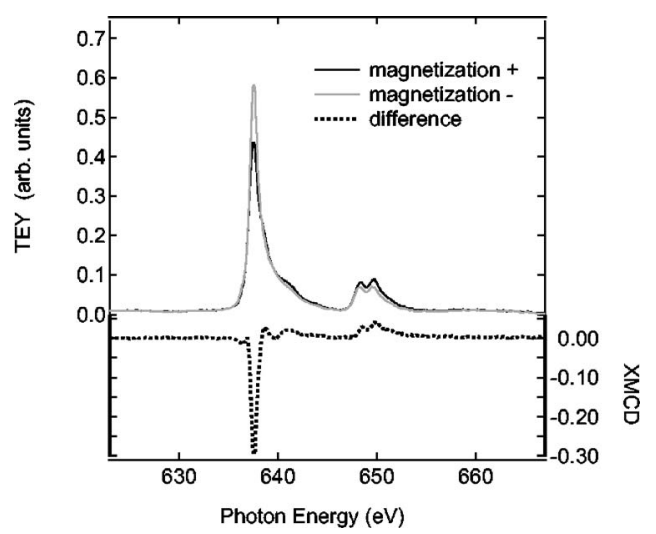

FIG. 3. Mn $L_{2,3}$ XMCD spectra and difference spectra (dotted line, multiplied by 2), at $17 \mathrm{~K}$, of sample $S 2$. The maximum asymmetry on the $L_{3}$ peak is $28.5 \%$, taking into account the light polarization $(70 \%)$ and the tilt angle of $45^{\circ}$. and corrected for light incidence angle and polarization, reaches its maximum of $28.5 \%$ at the $L_{3}$ edge, to be compared with over $50 \%$ obtained in Ref. 15 at $7 \mathrm{~K}, 99.9 \%$ circular polarization, and $2 \mathrm{~T}$ magnetic field applied along a hard magnetization axis. Taking into account the difference in temperature to compare our maximum dichroism with the one of Edmonds et al. in Ref. 15, we obtain a corrected value of $32 \%$. Note that also the SQUID loops shown in Fig. 1 show a reduced remanence of about $50-55 \%$ at $30 \mathrm{~K}$ in line with the XMCD results.

Figure 4 presents the evolution of the surface composition as a function of different sample treatment. The chemical etching produces a surface that contains $\mathrm{C}$ and $\mathrm{O}$, but with a Mn concentration below the detection limit. $S 3$ after $\mathrm{Ar}^{+}$ion sputtering shows a clear Mn $2 p$ signal with strongly reduced $\mathrm{C}$ and $\mathrm{O}$. The XAS spectral shape is very similar for $S 2$ and $S 3$, due to the higher probing depth of XAS with respect to XPS. Finally, in Fig. 4, we present the XPS spectrum from sample $S 3$ after $3 \mathrm{~h}$ long annealing at $200{ }^{\circ} \mathrm{C}$, in a oxygen pressure of $7 \times 10^{-7}$ mbar. In addition to the expected increase in the intensity of contaminants (O and $\mathrm{C})$, the Mn $2 p$ core level spectrum presents a clear energy shift of $1.5 \mathrm{eV}$ towards higher binding energies, characteristic of oxides. After annealing, we did not observe any XMCD signal, although the XAS spectral shape was within the error bar identical to the one measured on samples $S 1$ and $S 2$. Sample $S 3$ has been measured by SQUID magnetometry before and after the complete preparation treatment, revealing a drastic reduction of the remnant magnetization from about 50 $-55 \%$ to about $15 \%$ after sputtering, as shown in Fig. 1 . 




FIG. 4. XPS spectra of the sample $S 3$ at different steps of surface preparation: from bottom to top, $\mathrm{HCl}$ etched, sputtered (dashed line) and annealed in oxygen (continuous line). In the inset is visible the evolution of the Mn $2 p$ core level peaks, after background subtraction. A clear shift towards higher binding energy is observed in the $2 p$ line after annealing.

\section{DISCUSSION}

The observed $0.4 \mathrm{eV}$ shift towards lower binding energies in panel (a) of Fig. 2 is in good agreement with previous theoretical and experimental results, and it is ascribed to the additional screening made by the hopping of the $d$ electrons in the hybridized ground state. ${ }^{15}$ The comparison of various Mn compounds in Fig. 4 allows evaluating the evolution of the hybridization strength. Going from $\mathrm{Ga}_{x} \mathrm{Mn}_{1-x}$ As towards metallic Mn we observe an increased broadening of the spectra and a loss of multiplet features. Theoretical calculations by van der Laan reproduce the XAS spectra in a mixed ground state and indicate the increased hybridization of the Mn $3 d$ with conduction electrons (As $4 p$ in the case of $\left.\mathrm{Ga}_{x} \mathrm{Mn}_{1-x} \mathrm{As}\right)$ as the explanation of the experimental trend. ${ }^{15}$

The split structure at the $L_{2}$ edge was already observed in submonolayer of Mn on transition metals substrates, where a reduced coordination number favours strong hybridization of $d$ orbitals with the substrate, resulting in a correlation effect on $\mathrm{Mn} .{ }^{18-20} \mathrm{~A}$ change of the branching ratio with respect to its statistical value of $2 / 3$ has been already explained as a fingerprint of localization effect due to electrostatic interactions. $^{21}$ The measured increase of the branching ratio is directly proportional to the value of the Slater integrals, which are a description of the localization of the $d$ states. The data of Fig. 3 suggest that (i) the electronic structure of $\mathrm{Mn}$ in $\mathrm{Ga}_{x} \mathrm{Mn}_{1-x}$ As is markedly different from other III-V environment in which $\mathrm{Mn}$ is hosted. (ii) The Mn $3 d$ electrons in $\mathrm{Ga}_{x} \mathrm{Mn}_{1-x} \mathrm{As}$ are rather localized and, at this level of doping, Mn can be defined as a bulk impurity. The XPS data for the etched sample reveal a Mn-depleted layer at the surface and, keeping into account the different probing depth, the
XMCD signal we measured is truly representative of the bulk Mn. The split structure of the $\mathrm{Mn} L_{2}$ is, in the present case, a strong indicator of a reduced Mn-Mn overlapping in the bulk.

Other values of the XMCD asymmetry found in the literature are in the order of $39 \%$, measured in an applied field of $0.6 \mathrm{~T}$ (no post annealing), ${ }^{22}$ and of $55 \%$ (post annealed samples) in an applied magnetic field of $2 \mathrm{~T}$, perpendicular to the surface. ${ }^{15}$ Calculation performed by Ohldag et al. indicate that the maximum theoretical limit of XMCD is $59 \% .{ }^{12}$ Our experiment provides evidences of a net remnant magnetization in GaMnAs, in agreement with previous macroscopic measurements. ${ }^{23}$ Nevertheless the reduced XMCD value as compared to the theoretical limit indicates that some compensated or misaligned spins are present.

Our XMCD data at the Ga and As edges did not show any asymmetry, unlike what found by Keavney et al. ${ }^{24}$ who measured a weak asymmetry on samples with a higher Mn content $(x=0.07)$. The XAS spectrum of the sputtered sample is very similar to the one of the etched surface. After annealing in oxygen we observe only a small increase in the feature located at $541.2 \mathrm{eV}$, but no clear signature of a Mn oxide. With the support of the XPS data it is proved that the $\mathrm{XMCD}$ from the chemically etched sample is representative of the bulk magnetic properties of Mn embedded in the GaAs matrix, and that, on the opposite, the XMCD data measured in total electron yield mode on untreated samples, are affected by the surface oxides. Removing of Mn oxides by $\mathrm{HCl}$ etching leaves a $\mathrm{Mn}$-free, but $\mathrm{C}$ and $\mathrm{O}$ contaminated surface. Although Ar+ sputtering guarantees the removal of the surface oxide layer, an evident drawback of such a procedure is the possible surface damage and the selective sputtering yield.

Moreover, one has to take into account that $\mathrm{Ga}_{x} \mathrm{Mn}_{1-x} \mathrm{As}$ cannot be annealed up to the temperature needed to recover the surface stoichiometry $\left(560{ }^{\circ} \mathrm{C}\right)$ without affecting its crystal structure. ${ }^{25-27}$ The dynamics of the surface chemistry promoted by UHV annealing in oxygen atmosphere is clearly revealed by the XPS results of Fig. 4. The thermal process enables the diffusion of interstitial Mn, more mobile than substitutional one, towards the surface. Knowing that interstitial $\mathrm{Mn}$ is a double donor, its tendency to diffuse is drastically lowered when the environment is rich of oxygen, resulting in the formation of a stable oxides, as demonstrated by the $1.5 \mathrm{eV}$ shift in binding energy of the XPS spectrum corresponding to sample $S 3$.

\section{CONCLUSIONS}

Chemical etching and surface science cleaning methods in UHV were employed on different fragments of the same sample batches. Etching in $\mathrm{HCl}$ of GaMnAs and measuring in total yield mode gives reliable results on the bulk-diluted Mn since the surface is depleted of Mn oxides. We have shown that truly bulk-diluted Mn in GaMnAs shows remnant magnetization, by measuring XMCD at $17 \mathrm{~K}$. This result could be reached by exploring and understanding the relevance of the surface treatment of GaMnAs that is needed in order to warrant reliable XAS and XMCD data. 


\section{ACKNOWLEDGMENTS}

We thank D. Krizmancic for the acquisition and software support. We acknowledge J. Fujii and M. Marangolo for kindly letting us use part of their results on MnAs and MnPt. We gratefully acknowledge financial support by the Deutsche Forschungsgemeinschaft through the SFB 689.
${ }^{1}$ T. Dietl, H. Ohno, F. Matsukura, J. Cibert, and D. Ferrand, Science 287, 1019 (2000).

${ }^{2}$ S. A. Wolf, D. D. Awschalom, R. A. Buhrman, J. M. Daughton, S. von Molnár, M. L. Roukes, A. Y. Chtchelkanova, and D. M. Treger, Science 294, 1488 (2001).

${ }^{3}$ T. Dietl, H. Ohno, and F. Matsukura, Phys. Rev. B 63, 195205 (2001).

${ }^{4}$ H. Ohno, A. Shen, F. Matsukura, A. Oiwa, A. Endo, S. Katsumoto, and Y. Iye, Appl. Phys. Lett. 69, 363 (1996); G. Bouzerar, J. Kudrnovský, and P. Bruno, Phys. Rev. B 68, 205311 (2003).

${ }^{5}$ K. M. Yu and W. Walukiewicz, Phys. Rev. B 65, 201303(R) (2002).

${ }^{6}$ K. W. Edmonds, P. Bogusławski, K. Y. Wang, R. P. Campion, S. N. Novikov, N. R. S. Farley, B. L. Gallagher, C. T. Foxon, M. Sawicki, T. Dietl, M. B. Nardelli, and J. Bernholc, Phys. Rev. Lett. 92, 037201 (2004).

${ }^{7}$ S. J. Potashnik, K. C. Ku, R. Mahendiran, S. H. Chun, R. F. Wang, N. Samarth, and P. Schiffer, Phys. Rev. B 66, 012408 (2002).

${ }^{8}$ P. A. Korzhavyi, I. A. Abrikosov, E. A. Smirnova, L. Bergqvist, P. Mohn, R. Mathieu, P. Svedlindh, J. Sadowski, E. I. Isaev, Yu. Kh. Vekilov, and O. Eriksson, Phys. Rev. Lett. 88, 187202 (2002).

${ }^{9}$ M. B. Stone, K. C. Ku, S. J. Potashnik, B. L. Sheu, N. Samarth, and P. Schiffer, Appl. Phys. Lett. 83, 4568 (2003).

${ }^{10}$ K. W. Edmonds, K. Y. Wang, R. P. Campion, A. C. Newmann, N. R. S. Farley, B. L. Gallagher, and C. T. Foxon, Appl. Phys. Lett. 81, 4991 (2002).

${ }^{11}$ M. Malfait, J. Vanacken, V. V. Moschalkov, W. van Roy, and G. Borghs, Appl. Phys. Lett. 86, 132501 (2005).

${ }^{12}$ H. Ohldag, V. Solinus, F. U. Hillebrecht, J. B. Goedkoop, M. Finazzi, F. Matsukura, and H. Ohno, Appl. Phys. Lett. 76, 2928 (2000).
${ }^{13}$ S. Ueda, S. Imada, T. Muro, Y. Saitoh, S. Suga, F. Matsukura, and H. Ohno, Physica E (Amsterdam) 10, 210 (2001).

${ }^{14}$ Y. Ishiwata, M. Watanabe, R. Eguchi, T. Takeuchi, Y. Harada, A. Chainani, S. Shin, T. Hayashi, Y. Hashimoto, S. Katsumoto, and Y. Iye, Phys. Rev. B 65, 233201 (2002).

${ }^{15}$ K. W. Edmonds, N. R. S. Farley, T. K. Johal, G. van der Laan, R. P. Campion, B. L. Gallagher, and C. T. Foxon, Phys. Rev. B 71, 064418 (2005).

${ }^{16}$ K. W. Edmonds, G. van der Laan, A. A. Freeman, N. R. S. Farley, T. K. Johal, R. P. Campion, C. T. Foxon, B. L. Gallagher, and E. Arenholz, Phys. Rev. Lett. 96, 117207 (2006).

${ }^{17}$ see http://www.elettra.trieste.it/experiments/beamlines/

${ }^{18}$ H. A. Dürr, G. van der Laan, D. Spanke, F. U. Hillebrecht, and N. B. Brookes, Phys. Rev. B 56, 8156 (1997).

${ }^{19}$ Y. Huttel, C. M. Teodorescu, F. Bertran, and G. Krill, Phys. Rev. B 64, 094405 (2001).

${ }^{20}$ G. Fabricius, A. M. Llois, M. Weissmann, M. A. Khan, and H. Dreyssè, Surf. Sci. 331-333, 1377 (1995).

${ }^{21}$ B. T. Thole and G. van der Laan, Phys. Rev. B 38, 3158 (1988).

${ }^{22}$ I. T. Yoon, T. W. Kang, K. H. Kim, and D. J. Kim, J. Appl. Phys. 97, 063902 (2005).

${ }^{23}$ Y. L. Soo, G. Kioseoglou, S. Kim, X. Chen, H. Luo, Y. H. Kao, H. J. Lin, H. H. Hsieh, T. Y. Hou, C. T. Chen, Y. Sasaki, X. Liu, and J. K. Furdyna, Phys. Rev. B 67, 214401 (2003).

${ }^{24}$ D. J. Keavney, D. Wu, J. W. Freeland, E. Johnston-Halperin, D. D. Awschalom, and J. Shi, Phys. Rev. Lett. 91, 187203 (2003).

${ }^{25}$ U. Welp, V. K. Vlasko-Vlasov, X. Liu, J. K. Furdyna, and T. Wojtowicz, Phys. Rev. Lett. 90, 167206 (2003).

${ }^{26}$ J. Okabayashi, M. Mizuguchi, M. Oshima, H. Shimizu, M. Tanaka, M. Yuri, and C. Chen, Appl. Phys. Lett. 83, 5485 (2003).

${ }^{27}$ T. Hayashi, M. Tanaka, T. Nishinaga, H. Shimada, H. Tsuchiya, and Y. Otuka, J. Cryst. Growth 175, 1063 (1997). 